\section{L'ARDOISE ET SON EXPLOITATION}

\section{I. - CONSIDÉRATIONS GÉNÉRALES.}

L'industrie ardoisière a subi de nombreuses vicissitudes et, si les conditions économiques du marché en ont été le facteur principal, si l'universelle loi de l'offre et de la demande est venue plusieurs fois paralyser l'activité de cette industrie jadis florissante et la faire tomber dans le marasme, d'autres causes aussi intervinrent, dont la plus importante était naturellement le prix de revient relativement élevé de l'ardoise.

D'autre part, faute de machines on n'utilisait que bien peu une partie fort importante du schiste abattu et l'on faisait passer en un formidable déchet des plaques plus ou moins grandes qu'on ne pouvait utiliser.

Le perfectionnement graduel des appareils à vapeur fit bien par contre coup étudier de plus près les machines à abattre, extraire ou travailler l'ardoise, mais il a fallu l'intervention de l'électricité pour déterminer l'essor auquel nous commençons à assister aujourd'hui. Jusqu'à ces dernières années on n'avait songé, pour tous les services de l'exploitation d'une ardoisièrc, à n'utiliser que sur une petite échelle les incomparables avantages de l'électricité. Naturellement on commença par l'éclairage; puis on risqua quelques timides transports de force pour actionner de petites pompes d'épuisement; c'est à perne si l'on essaya quelques perforatrices.

En réalité, tout ou presque tout est resté à la vapeur avec ses multiples inconvénients. Mais comme toujours, comme partout, la houille blanche est venue bouleverser tout cela.

Lorsque sous l'influence d'une meilleure fabrication mécanique, l'industrie ardoisière put livrer au commerce des produits plus choisis, plus homogènes, plus finis, lorsqu'elle put, en toute sécurité contracter des engagements, signer des marchés considérables de tel modèle toujours identiquement le même, la demande s'accrut dans des proportions fantastiques ct devant la facilité d'emploi, la propreté et l'extrême durée de l'ardoise, son emploi se généralisa partout. Chose curieuse, il se déreloppa précisément surtout dans les pays qui ne contenaient pas de schistes ardoisiers jusqu'ici exploitables. C'est alors qu'en France, par exemple, où il y a un peu partout des gisements de cette nature; on se mit à les utiliser. Ils étaient tout d'abord inexploitables, par suite principalement du haut prix du combustible joint à l'élévation du prix de son transport.

En effet, en pays montagneux (et c'est presque toujours ainsi pour une ardoisière), le prix du cheval-heure peut atteindre des taux absolument prohibitifs. En le calculant, pratiquenent, commercialement, on arrive facilement à o fr. 50 et même plus.

On conçoit facilement que, dans de telles conditions, aucun gisement ardoisier n'était susceptible de donner des bénéfices. Aussi laissait-on le schiste là où il était.

Tout est changé maintenant que l'utilisation des forces motrices naturelles permet, comme on le sait, d'avoir le kilowattheure livré à plus de 50 kilomètres à des prix variant de ofr. 10 centimes à ofr. 05 , par grandes quantités.

Qu'arrive-til devant un tel résultat? C'est que dès qu'une société se forme pour l'exploitation d'un gisement ardoisier, elle se préoccupe tout d'abord de l'existence à proximité, d'une chute d'eau qu'elle puisse utiliser. On consacre, purement et simplement, une certaine somme qui, rarement, a besoin d'être importante, soit à l'achat d'un moulin qu'on améliore, soit à l'acquisition d'une chute non utilisée qu'on aménage pour son maximum de puissance, soit à tout autre travail de captage, de telle façon que ces frais de premier établissement étant faits, on n'ait plus à porter en dépenses le combustible quotidien, ni surtout l'entretien formidable que nécessitent générateurs et moteurs à vapeur, à alcool, péfrole ou essence.

Il nous a été donné, à nous-même, d'étudier et d'utiliser pour une ardoisière, un vieux moulin, donnant par un petit barrage, une chute d'eau de I mètre de hauteur à peine, et dont le débit varie de I mètre cube pendant les deux mois de sécheresse à 3 mètres cubes au moins pendant les 10 au. tres mois.

Quelle que faible que soit l'énergie en houille blanche, dont on pourra disposer dans le voisinage d'une ardoisière, on devra toujours l'utiliser. C'est grâce à elle que cette industrie sce relève actuellement.

L'inaltérabilité de l'ardoise, à l'air et à l'humidité, l'a fait employer depuis le $\mathrm{X}^{\mathrm{e}}$ siècle environ, pour la couverture des édifices.

Suivant la force du vent dans le pays, on peut employer des ardoises plus ou moins grandes. Dans les points peu exposés au vent, on préfère souvent la dimension maxima, dite Grande Anglasse, qui coûte plus cher mais est à peu près inusable.

On l'utilise, en outre, pour les carrelages et revêtements de salles de bains, de latteries, pour la fabrication des mangeojres des écuries, des tablettes de cheminées, des tables de billard, des isolateurs électriques et autres usages bien connus.

Depuis qualque temps, son emploi tend à se généralıser de plus en plus, surtout depuis que les nouvelles machines qu'on emploie ont permis de présenter un produit fini, poli et totjours de même dimension.

L'ardoiserie notamment (objets diver's fabriqués) a pris un essor considérable.

Ce fait a permis de pousser l'exploitation des anciennes as doisières et d'en ouvrir de nouvelles, auxquelles on ne songeait pas il y a quelques amnées.

Il est à prévoir que la demande, sans cesse croissante, ne s'arrêtera pas de sitôt. C'est pourquoi tout propriétaire de gi. sements de cette nature a le plus grand intérêt à savoir sill peut, ou non, les mettre en exploitation.

Quelques notes à ce sujel ne seront pas déplacées dans the revue telle que celle-ci.

\section{II. - PROSPECTION ARDOISIÈRE.}

Le silicate d'alumine auquel on a donné le nom de schiste ardoisier est une roche sédimentaire ̀̀ structure foliacée extrémement répandue sur la surface du globe.

C'est, en réalité, un schiste argileux impur et qui est plus ou moins fissile, dont la couleur varie du gris violacé ou bleuấtre au gris rouge. Lorsqu'il l'est suffisamment, on en fabrique les ardoises de toutes dimensions et de toutes épaisseurs.

Le schiste ardoisier étant une roche qui doit sa propriété essentielle à une action métamorphique, ne peut exister que là où cette action a eu tout son développement, sur de puissants dépôts argileux, c'est-à-dire dans les terrains très anciens de la croûte terrestre.

On le trouve en abondance, alternant avec des grès et des quartzites, dans certaines contrées remontant aux premiers âges géologiques et qui se signalent par les dislocations et les contournements que les couches ont subies.

Le plus souvent le schiste a une texture grossière, il n'est alors divisible qu'en plaques assez épaisses ou en feuillets de faibles dimensions.

Pour qu'il puisse donner ces lames minces et résistantes, qu'on emploie dans la couverture des maisons il faut quil possède un ensemble de propriétés rarement réunies et, en particulier, un grain fin, homogène, compact.

La fissilité ou schistosité, il est important de le remarqué; est indépendante du plan de la stratification, en sorte quele plan du feuillet et celui de la couche font généralement entre eux un angle plus ou moins prononcé ( $I$ ).

Les gisements ardoisiers se présentent presque exclusivement sous forme de veines plus ou moins puissantes, régulit. res et fissiles. La France est particulièrement bien parfaget

(1) Watrin. Les Ardoisières des Ardennes. 
sous ce rapport, et ses ardoisières du bassin d'Angers, des Ardennes, de la Savoie et des Pyrénées sont connues du monde entier par les centaines de millions de produits qu'elles ont exporté.

Dans ces différents bassins, les couches sont presque verticales, comme à Angers et en Bretagne, où l'on trouve des couches très rapprochées les unes des autres et peu épaisses, de $0 \mathrm{~m}$. 50 à I mètre.

Quant au gisement d'Angers, il est remarquable par l'existence presque exclusive des schistes en veines énormes, à l'envers de ce qui se voit aux Ardennes où des couches de 4 à 15 mètres ne sont pas rares, mais où des bancs de quartzites alternent avec des couches schisteuses.

De mêrre en Savoie et dans les Pyrénées où l'on trouve des séries de veines schisteuses puissantes, formant quelquefois des contreforts entiers de montagne. Mais rarement alors toute la veine est fissile. En réalité, ce sont des schistes ardoisiers et non de l'ardoise proprement dite.

Le passage du schiste non fissile à l'ardoise y est généralement graduel, surtout lorsque l'on s'enfonce; la teinte change également et l'on peut alors suivre une veine utilement exploitable ou kien exploiter une sorte de véritable poche de dimensions quelquefois considérables qui se termine en coin des. des deux côtés, survant la direction.

L'exploitation est alors singulièrement compliquée et à une période de beaux bénéfices résultant de l'abatage d'une telle partie du gîte, succède une période onéreuse où les découverts, les déblaiements ou la recherche de la bonne couche viendiont grever lourdement le budget. Il faut, dans ce cas, organıser le travail pour une exploitation suivie et se garder de dépiler ce que l'on voit de beau. On tomberait d'abord dans les pires erreurs au point de vue de l'appréciation même de la richesse du gisement et, au bout de peu de temps, on serait obligé de procéder à nouveau à des travaux de mise en valeur en bouleversant les anciens. Résultat : Grosses dépenses et perte de temps très préjudiciables.

Les gisements ardoisiers qui peuvent s'exploiter à ciel ouvert sont naturellement favorisés sous ce sens, bien que le découvent soit parfois difficile et souvent très onéreux. Mais toutes les fois qu'on peut le faire, il ne faut pas hésiter. C'est ce qui se passe à Angers et dans certains gisements des Pyrénées. J.orsque les couches sont peu épaisses et verticales, ou à peu près, ce découvert ne peut être opéré et l'exploitation est plus délicate. Enfin, lorsque les couches sont inclinées de 25 à $45^{\circ}$, comme dans les Ardennes, et que la fissilité ne commence qu'à partir de 50 ou 100 mètres de la surface (ou bien lorsqu'on est obligé d'aller à cette profondeur pour trouver la veine), on opère par descenderies.

Nous développerons ce sujet au paragraphe 6 .

\section{III}

En présence de ces immenses dépôts que forment les schistes plus ou moins ardoisiers, de la facilité de les reconnaître, de leur localisation dans les terrains siluriens et cambriens, il semble au premier abord qu'une prospection ardoisière soit la plus simple du monde.

Il est loin d'en être ainsi. L'Ingénieur aura, au contraire, dans bien des cas, beaucoup de difficultés à donner un avis sans réserves, ce qu'il voit n'étant bien souvent qu'un trompe l'oil naturel.

Tout d'abord, ces couches schisteuses, ces phyllades, ne sont pas, en général, visibles à l'œil nu.

Mais la reconnaissance d'un terrain ardoisier ou non est des plus simples, par suite de la nature même des éléments qui le composent dès qu'on peut les apercevoir.

Lorsque les schistes viennent affleurer, on voit immédiatement au-dessous d'une épaisseur plus ou moins grande de terre végétale, une couche de matériaux détritiques, la plupart du temps assez décomposés, formée de débris d'ardoises généralement menus, disposés pêle-mêle, sans aucune stratification, absolument-comme si on était en présence d'une décharge publique.

Au-dessous, vient ce qu'on appelle "engaize " dans le Nord, "cosses" dans le Midi et l'Ouest. C'est déjà un schiste moins détritique où la stratification est bien apparente et vient généralement en discordance avec la couche de débris dont nous venuns de parler.

Le schiste est encore très fendullé, très brisé cn lamelles plus ou moins minces, tantòt collées les unes aux autres, tantôt un peu distantes. De plus, sous l'influence des agents atmosphériques ou de l'eau, l'oxydation des particules ferreuses a, dans la plupart des cas, formé une couche rougeâtre, d'une teinte de rocaille, sur toute la surface de ces morceaux brisés et il faut les casser pour apercevoir le grain noir ou bleuté de l'ardoise.

Cette " engaize ", ces " cosses " sont naturellement inutilisables. Lorsqu'on les rencontre, on est certain de trouver un peu plus bas de l'ardoise fissile, mais l'inclinaison des bancs exploitables n'est généralement pas celle des couches supérieures décomposées. Il importe d'y faire la plus grande attention pour les travaux futurs Ces " cosses " atteignent souvent des épaisseurs considérables de 15 à $18^{\mathrm{m}}$. Leur existence est en même temps un obstacle à la reconnaissance du gisement ardoisier pouvant exister en dessous.

Presque toujours les phyllades suivent les allures tourmen. tées des dépôts qui les enclavent et on ne peut guère les observer que sur les escarpements ou les flancs des vallées; presque (partout ailleurs leurs affleurements sont masqués par des dépôts modernes atteignant quelquefois une grande épaisseur et par la végétation.

Il ne faut pas croire, d'ailleurs, qu'une couche phylladienne ait dans toute son étendue les mêmes qualités. Elle est au contraire sujetle à de fréquents caprices : tantôt elle peut devenir dure au point de ne plus se laisser fendre, tantôt elle est découpée par de nombreuses fentes qui réduisent le phylladc en petits fragments, tantôt elle est brouillée ou altérée ou sans aucune consistance; enfin il se peut que sous l'effet des dislocations et des compressions elle ait été redressée, plissée, contournée, amincie, renforcée, repliée, etc. etc.

Il en résulte qu'à une partic de la couche où la pierre donnc d'excellentes ardoises en succède fréquemment une autre qui n'a aucune valeur.

Dans un même gisement ardoisier il peul y avoir ainsi, et il y a la plupart du temps (pour ne pas dire toujours) des parties du même banc qui sont fissiles el d'autres qui ne le sont pas. En outre, il existe toujours de nombreux bancs intercalaires noirs non ardoisiers ou de roches schisteuses ou quartzeuses.

Ces bancs quelquefois bien visibles, par ailleurs ne le sont pas du tout. A première vue, on peut tout prendre pour dc l'ardoise, et non seulement cuber d'une manièrc fort erronće, mais encore ces bancs intercalaires peuvent, dans certains cas, entraver tellement l'exploitation qu'elle deviendrait onéreuse.

Le prospecteur habile passera donc le temps nécessaire à décaper une largeur suffisante du banc ardoisier, le tout même, à essayer la fissilité à l'aide d'outils appropriés et il portera tout spécialement son attention sur le nombre, la disposition, l'importance, la manière de se poursuivre des bancs intercalaires. Il devra toujours se dire que le schiste ardoisier, même paraissant constituer une veine ou une masse homogène, n'est jamais avantageusement exploitable dans toutes ses parties, et que ces injections de quartz ou de calcaire ou bien des fentes très rapprochées qu'il ne voit pas, le rendent impropre à toute fabrication de l'ardoise.

Or, on est très facilement porté à s'illusionner sur la régularité de composition et d'allure d'un banc ardoisier par la partie plus ou moins considérable que l'on en voit.

Il semble à première vue que ces couches peu tourmentées en un point doivent se prolonger sous le même aspect, avec 
les mêmes propriétés physiques et chimiques sur de grandes étendues.

C'est le contraire presque toujours et l'irrégularité est la règle de l'allure des bancs ardoisiers. Il faut donc compter avec cela lors de l'estimation ou de la contenance du gisement.

Il n'y a aucune loi qui préside à la distribution de ces accidents et qui permette de dire, par conséquent, dans quelle proportion se trouve le schiste fissile par rapport à la matière utilisable.

Dans n'importe quelle ardoisière, on trouve des régions plus ou moins étendues dans laquelle le schiste devient inexploitable. Rien de plus délicat à déterminer. Les défauts qui suppriment les propriétés industrielles, sont parfois très peu apparents et peuvent surgir, d'une manière tout à fait inattendue, sans que rien ne les fasse deviner à l'homme le plus expérimenté.

Aussi y a t-il peu d'industries minérales plus aléatoires que celle de l'ardoise, car l'expérience la plus consommée est souvent impuissante à faire prévoir ces variations de nature du schiste qui peuvent se produire d'une manière tout à fait inattendue (I).

11 est très rare, nous l'avons déjà dit, qu'on ait à prospecter un gisement ardoisier à ciel ouvert.

En affleurement, les constatations sont naturellement plus faciles et, notamment, on déterminera de suite la position et l'importance du dépôt ardoisier.

Il faudra alors s'attacher à reconnaître la continuité et l'homogénéité des dépôts et l'on verra de suite si l'ardoise peut être exploitée à ciel ouvert ou souterrainement, c'est-à-dire si $1_{\imath}$ qualité du schiste est suffisante en affleurement pour cela.

Presque toujours, en effet, les affleurements ont été altérés par les eaux et les agents atmosphériques. Ce n'est qu'à une profondeur ordinairement supérieure à 30 mètres que le schiste commence à acquérir la dureté nécessaire à la résistance de l'ardoise. Cependant il existe des affleurements exploités à ciel ouvert où la bonne ardoise se trouve pour ainsi dire à la surface.

Plus généralement, ils sont recouverts par la partie décomposée qu'on appelle la " cosse " ou l' "engaize ", qu'il faut déblayer pour exploiter à ciel ouvert et dont nous avons déjà parlé. Lorsqu'aux affleurements l'épaisseur des "cosses " est peu considérable, seulement de quelques mètres par exemple, il est évident que c'est un bonne chose. L'exploitation du gîte sera naturellement moins onéreuse puisque, d'une part, on aura peu de découvert à manier et que, de l'autre, on atteindra de suite la bonne ardoise.

En tous cas on ne peut jamais savoir à première vue, soit en affleurement, soit dans une mine souterraine d'ardoise si le schiste sera fissile; c'est l'exploitation seule qui le dira.

C'est naturellement de cette qualité primordiale, la fissilité, que dépend la prospérité du gisement. Souvent elle est très marquée aux affleurements et disparaît quand l'exploitation a atteint une certaine profondeur. Dans ces conditions, la couleur du schiste a été modifiée et la pierre a acquis un reflet cristallin un peu rougeâtre qui lui a fait donner le nom de pierre gelée (2).

Mais on peut cependant par l'observation générale du dépôt, du plissement, par la composition et la nature des couches, se former une opinion qui doit se rapprocher de la réalitć.

Ce qu'il y a déjà de certain, c'est que la qualité des ardoises augmente toujours avec la profondeur.

Mais, d'un autre côté, si l'on a la chance d'avoir dès la surface du schiste non décomposé, cela vaut évidemment mieux.

On comprend, en effet, qu'il est coûteux de traverser, comme à Fumay par exemple, Ioo mètres de quartzites, terrain extrê-

1) Nivoit. L'Ardoise. - (2) Watrin, Op. cit. mement dur, d'où immobilisation considérable pour le fonçage du puits inclıné et ensuite frais d'exploitation plus ćlevés.

D'un autre côté, une ardoisière aura d'autant plus de chan. ces d'être prospère qu'on y pourra fabriquer des plaques de plus grande dimension (le prix de vente des dalles d'ar. doise est 4 fois plus élevé que celui des ardoises de couverture), cette condition marchant de pair avec la fissilité des couchë de manière à pouvoir fabriquer aussi des ardoises ordinaires.

Elle aura également d'autant plus de valeur que les cou. ches auront une allure moins tourmentée, ne seront pas redres. sées, fracturées par des failles ou trop séparées, comme il va être dit, par des couches de cailloux ou de quartz ou qu'elles ne soient pas divisées par de nombreux joints qui rendent quelquefois la pierre absolument impropre à toute fabrication. De même lorsqu'on a des couches épaisses et homogènes, on a naturellement un pourcentage de déchet beaucoup moindre et une bien plus grandc facilité pour extraire de grandes pièces.

On voit donc que s'jl r'est pas difficile de reconnaître un schiste ardoisier, c'est-à-dire un gisement propre à donner de l'ardoise, il est, par contre, très délicat de se prononcer sur sont exploitabilité.

Un premier élément de constatation qui, du coup, élimine toute autre recherche consiste, ainsi que nous l'avons déjà dit, dans la localisation de ces schistes dans les terrains primaires. Par conséquent, lorsqu'on aura déterminé la présence de l'ardoise en affleurement par l'ardoise elle-même ou souterrainement par les "cosses ", on s'appliquera à l'étude des plissements et des failles de la contrée qui donnent la plus utile indication sur la continuité probable du gîte.

L.es " cosses " sont, le plus souvent, rongées par l'oxyda. tion de la pyrite qu'elles contiennent. Souvent même de petites mares se forment, pleines d'eau ocreuse. S'il y a beaucoup de pyrite, on le reconnaît à cette coloration et c'est déjà un mauvais point pour la valeur du dessous, bien qu'il ne faille pas oublier qu'en général la pyrite existe surtout aux afflurements

Dans un pays manifestement schisteux et où des couches d'ardoises sont déjà connues et exploitées, il peut être très intéressant de chercher le prolongement de ces couches, qui peuvent fort bien s'être trouvées dérangées par des failles et ne pas exister là ou l'on croit les rencontrer avec le plus de chances de succès.

Le meilleur procédé consiste alors à faire des sondages, à les developper et à les pousser assez loin pour être sûr d'arriver au-delà du massif rocheux, dans lequel sont intercalées les veines d'ardoise connues et exploitées par ailleurs.

C'est un procédé d'une certitude absolue, mais très cher.

Une prospection ardoisière ne présente, en général, aucun fait saillant. La fatigue et les dangers sont les mêmes que dans toute mission de mine, peut-être à un degré un peu plus élevé, les promenades en terrain silurien et cambrien n'étant pas toujours commodes et, d'autre part, les anciennes exploitations qu'on est appelé à visiter, étant peu solides.

Ici, en effet, on se trouve toujours en présence d'ouvrages exploitćs aux dimensions généralement considérables, oì aucune surveillance ni entretien n'ont eu lieu depuis nombre d'années. L'exploitation ancienne a souvent laissé des masses schisteuses collées au toit et qui peuvent se détacher à chaque instant, et la dimension des vides par rapport aux piliers est des plus inquiétantes. De plus nous n'avons pour notre part, jamais visité un gisement de ce genre sans le trouver plein d'eau... tout au moins la première fois que nous y étions appelé. Signalons enfin un fait pratique qui a bien son importance.

Lorsqu'on va visiter une ardoisic̀re déjà reconnuc ou exploitée antérieurement et qu'il s'agit de reprendre, on peut être sûr que le vieil ouvrier qui vous y mènera, vous conduira aux 
parties saines naturellement... selon lui, c’est-à-dire, en réalité, anx plus mauvaises du gîte.

Il est guidé lui-même en cela par deux instincts : d'abord, les méthodes d'exploitation étant restées stationnaires depuis le moyen âge, ce sont encore les moyens les plus primitifs qui sont employés et l'on va presque toujours au hasard pour le dépilage.

Le vieil ouvrier qui souvent a été le directeur de l'ancienne exploitation (c'est le nom qu'en langage ardossier on donne à un ouvrier plus intelligent que les autres, connaissant la carrière ct ayant rempli le rôle de contremaître) n'a que les connaissances les plus restreintes au sujet d'une exploitation rationnelle et n'opère que par la légende de la manière d'opérer transmise de père en fils, c'est-à-dire par traditions. Il peut donc être de bonne foi et considererce qu'il vous montre comme une partie bonne du gisement, alors que cela ne vaut rien. 11 est donc imprudent de s'en remettre à ses dires sans vérifier. Ensuite, et c'est alors un phénomène très naturel, très humain, quoique et peut-être pour cela indélicat, pour ne pas dire plus, il ne faille pas oublier que l'on se trouve en présence du paysan amant par dessus tout sa terre, son bien, dont il ne peut se séparer, même après l'avorr vendu.

11 peut donc arriver que l'on fasse attaquer exprès dans la partıe la plus mauvaise, dans le pourri, de manière à pouvoir acquérir plus tard à bas prix le tréfonds, lorsque la Compagnie sera ruinée ou bien pour passer l'affaire à une Société voisine.

Quant à l'appréciation du tonnage, elle sera relativement plus facile que pour une mine métallique en général. Mais il y aura lieu de tenir compte des partıes déjà exploitées, du déchet d'exploitation, des piliers abandonnés, des serrées, des portıons de veine non fissiles, des banrs de grès intercalaires, des diverses galeries à établir, des éboulements, des pourris, des déchets de fabrication et d'une partie inexplontable du gite située près de sa. surface.

Sans compter les ouvrages déjà exploités, l'ensemble de ces déchets monte à environ $75 \%$.

Mais nous devons insister encore davantage sur cette question des déchets.

Si c'est une nouvelle ardoisière qu'il s'agit d'ouvrir dans un gisement souterrain, presque toujours l'Ingénieur trouvera la première prospection déjà faite. Quelques tranchées pas très profondes, une ou deux petites descenderies, quelquefois un travers bancs ou un puits peu profond auront déjà montré la possibilité ou même la présence certaine du schiste fissile en un point déterminé.

Rarement ces travaux auront donné lieu à d'importants déblais. Mais il n'en est pas de même lors de la reprise d'une ancienne ardoisière exploitéc au temps jadis et abandonnée pour une cause quelconque.

Là, avec le marché actuel si florissant de l'ardoise, il est permis d'avoir une moins mauvaise idée du gisement parce qu'on n'en a pas-poursuivi l'exploitation.

L'Industrie de l'ardoise a subi des fluctuations telles que les prix de vente à l'époque sont peut-être l'unique cause de l'abandon de l'affaire. Puis la question éternelle des transports intervient aussi. Faute de moyens de communication, il est fort possible que les ardoises ne pouvant s'exporter, on soit arrivé vite à "faire le plein " dans le pays; enfin, et c'est presque toujours la vraie cause, on a dû abandonner parce que, travaillant au jour le jour, avec des moyens insuffisants, aucun matériel de hissage ou de pompage, l'exploitation était arrivée en profondeur à un niveau tel qu'il devenait trop dispendieux d'enlever et de rencontrer la pierre à travailler et surtout les déblais, bien qu'on en laissât une bonne partie dans les travaux du fond.

Les veines d'eau à cette profondeur ont dû être également une cause d'abandon et l'on est encore, à l'heure actuelle, tellement habitué à l'exhaure de l'eau par le travail humain, consistant à remplir des tonneaux avec des seaux et à les remon- ter dans un wagonnet tiré par un treuil à bras, qu'il y a des équipes de mineurs qui ne font que cela un jour par semaine, pour " dénoyer " les travaux. Inutile de dire que ce non sens tend à disparaître fort rapidement.

Quoiqu'il en soit, on se trouve alors en présence d'une quantité énorme de déblais. Et c'est là que l'Ingénieur a besoin de toute sa finesse, de toute son expérience et de ses qualités spéciales d'observation pour estimer si, dans les limites du champ d'exploitation qu'il a à considérer, le cube restant à exploiter n'est pas réduit à une portion infime - ou trop coûteux à atteindre.

D'une part, le cube des déblais en vue, en tenant compte du foisonnement; de l'autre, celui des tas plus ou moins considé rables restés dans les travaux (ceux-ci étant presque impossibles à cuber); enfin, la portion qui a pu en être enlevée pour des remblayages, des constructions de routes, etc., serviront à estimer ce qui a pu être enlevé. Par comparaison avec la puissance de la couche ou des couches, on aura donc une idée approximative de ce qui reste.

Il est donc, plus que dans une mine métallique, fort important de porter la plus grande attention sur ces dechets empilés.

\section{V}

Les bancs de bonne ardoise sont toujours séparés par des dépôts plus ou moins importants de quartz, quartzites ou grès appelés crapés dans les Ardennes, marbre dans les Pyrénées.

A Angers, les schistes ardoisiers forment au milieu des autres schistes plusicurs couches appelées icines de puissance variable, mais généralement assez considérable et dépassant par endroits Ioo mètres. Sur une pareille épaisseur, la qualité du schiste n'est pas toujours la méme, l'ardoise y est plus ou moins fissile. Par exemple, absence presque absolue de bancs de grès intercalés ( $I$ ), tandis que dans la plupart des cas, ces bancs existent plus ou moins épais, séparant les couches exploitables. C'est ainsi que, dans les Ardennes, on trouve des couches d'ardoise de 25 à 30 cent. d'épaisseur, mais surtout des veines de 0.25 à 2 mètres, séparéespár des lits de grès. ieaucoup de petites veines intercalaires ne sont pas explortables.

Au contact de ces bancs et sur une épaisseur qui peut varier de I centimètre à 20 , le schiste ardoisier s'est souvent trouvé métamorphisé et inexploitable, mais ce léger accident est rarement assez important pour avoir une influence sérieuse sur la qualité du gîte. Quelquefois aussi ces injections quartzeuses se ramifient, se divisent et disparaissent.

Les mineurs des Pyrénées, dans leur langage imagé, disent que ce marbre est le père de l'ardoise, parce que, à l'avancement, ils rencontrent d'abord le schiste métamorphisé impropre à la fabrication des ardoises, pius le marbre, puis encore une couche de schiste mauvais et, enfin, ils retrouvent une veine 'd'ardoise d'excellente qualité. En réalité, jamais de bonne ardoise sans marbre auparavant.

\section{V. - QUALITÉ d'UNE BONNE ARDOISE.}

Le schiste ardoisier est presque exclusivement formé de silice et d'alumine. Ces deux substances forment les $3 / 4$ de sa composition. Ine ardoise est d'autant plus dure qu'elle contient plus de silice, et c'est la plus ou moins grande proportion de ces deux éléments, silice et alumine, qui détermine de la façon la plus importante la qualité bonne, médiocre ou mauvaise de l'ardoise considérée.

Le reste est formé par du titane, du péroxyde de fer, de la magnésie et de la chaux.

Le tableau suivant sera très utile à consulter, car il donne la composition de types d'ardoise d'excellente qualité dont ne

(1) Fuchs et Delaunay. - Traité de gites minéraux et métalli- 
devront pas s'éloigner les ardoises que l'Ingénieur peut avoir à examiner.

Les couleurs des ardoises, c'est-à-dire des schistes dont on les extrait, sont très variées; on admet qu'une ardoise est d'autant meilleure que sa couleur est plus foncée. On en trouve avec des nuances rouge, lie de vin, violette, bleue, grise, verte, noire, noire bleutée. Une couche de schiste change quelquefois brusquement de couleur sans qu'il y ait aucune solution de continuité dans la pierre. Sa principale qualité au point de vue de la fabrication dos ardoises est de présenter un clivage facıle, d'être très fissile et d'avoir une cassure nette.

Les schistes quoique bien fissiles, peuvent être chargés plus ou moins de pyrites de fer el autres corps hétérogènes et accidentels, désignés par les ouvriers sous les noms de lamproie, mouche, blanc, qui interrompent la fente et occasionnent dans la fabrication un plus grand déchet, de façon à rendre telle portion de veine avantageusement exploitable et telle autre absolument pas. qui consiste à l'immerger verticalement dans l'eau, de manière qu'elle n'y plonge que jusqu'au tiers de sa hauteur. Si au bout de 24 heures environ l'extrémité supérieure est parfaitement sèche, l'ardoise sera jugée bonne et de compacité suffisante : dans le cas contraire elle devra être rejetée, l'ascension dé l'eau dans la masse en démontrant la porosité (I).

Une bonne ardoise pour être marchande, doit posséder de nombreuses qualités.

Elle doit être bien plane, à grain fin, dure, sonore, pesante, résistante à l'écrasement, compacte, avoir une structure homo. gène et serrée, une couleur foncée uniforme agréable à l'œal, une épaisseur régulière, ne pas présenter d'aspérités mais, ał contraire, avoir une surface bien lisse, naturellement sans escaliers, cascades, échelons, esquilles, et ne pas trop contenir de pyrite de fer ou de cristaux de fer magnétique.

Ces grains qui sont noirs et de forme octaédrique sont le plus souvent allongés suivant une ligne à laquelle on a donné le nom de Longrain dans les Ardennes.

Tableau de la Composition des types d'Ardoise francaise

\begin{tabular}{|c|c|c|c|c|c|c|c|c|c|c|c|c|c|}
\hline \multirow{3}{*}{$\begin{array}{l}\text { COMPOSITION } \\
\text { DES ARDOISES }\end{array}$} & \multirow{3}{*}{ 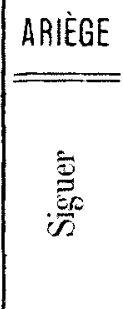 } & \multicolumn{2}{|c|}{ SAVOIE } & \multicolumn{2}{|c|}{ ANJOU } & \multirow{3}{*}{ 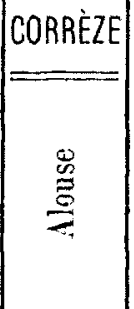 } & \multicolumn{7}{|c|}{ ARDENNES } \\
\hline & & \multirow{2}{*}{ 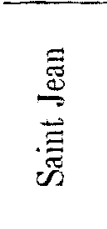 } & \multirow{2}{*}{ 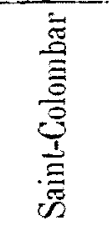 } & \multirow{2}{*}{$\frac{\overrightarrow{\underline{D}}}{\stackrel{\underline{Z}}{E}}$} & \multirow{2}{*}{ 莺 } & & \multicolumn{3}{|c|}{ Rimognes } & \multirow{2}{*}{ 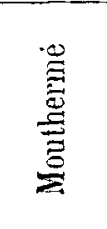 } & \multicolumn{2}{|c|}{ Fumay } & \multirow{2}{*}{ 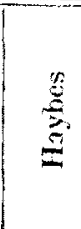 } \\
\hline & & & & & & & $\begin{array}{c}\text { Arduise } \\
\text { bleue }\end{array}$ & $\begin{array}{l}\text { Ardense } \\
\text { grenue } \\
\text { grise }\end{array}$ & $\mid \begin{array}{c}\text { Ardose } \\
\text { grenue } \\
\text { fine verte }\end{array}$ & & $\begin{array}{l}\text { Scliste } \\
\text { rouge }\end{array}$ & $\begin{array}{c}\text { Schiste } \\
\text { vert }\end{array}$ & \\
\hline Siltce ....... & 68.00 & 32.25 & 48.95 & 50.25 & 48.60 & 87.30 & 61.00 & 60.30 & 60.00 & 59.91 & 61.57 & 65.42 & 68.0 \\
\hline Alumine...$\ldots \ldots$ & 13.00 & 15.00 & 18.89 & 29.68 & 23.50 & 3.20 & 9.60 & 12.00 & 10.80 & 19.51 & 19.22 & 19.98 & 13.0 \\
\hline Protoxyde de fer & 9.00 & 11.00 & 3.24 & 7.57 & 11.30 & 4.80 & 12.00 & 11.00 & 12.30 & 2.74 & 6.63 & 3.08 & 6,8 \\
\hline Magnésie.. & 1.2 & 0.25 & 1.05 & 1.95 & 1.60 & 1.15 & 4.80 & 4.90 & 8.80 & 2.35 & 2.00 & 1.49 & 1.00 \\
\hline Chaux ........... & 0.7 & 29.50 & 12.05 & 0.53 & 0.00 & 0.8 & 3.30 & 2.80 & 2.60 & 0.40 & 0.22 & 0.23 & 0.00 \\
\hline Eau $\ldots \ldots \ldots \ldots \ldots$ & 4.6 & 19.00 & 11.85 & 5.88 & 7.60 & 2.75 & 3.80 & 3.90 & 4.00 & 3.46 & 3.25 & 3.11 & nen dose \\
\hline Potasse et Soude. & 3.0 & non dosé & 2.50 & 4.16 & 4.70 & non dosé & 4.80 & 4.90 & 6.20 & nen dosé & non dosé & non dose & nos dos: \\
\hline Titane & non dosé & non dosé & non dosé & non doséé & nou dosé & non dosé & non dosé & non dosé & non dosé & 1.46 & 1.31 & 1.15 & 1.0 \\
\hline
\end{tabular}

C'est ce qui explique les nombreux échecs éprouvés par les anciens exploitants d'Angers par exemple, qui, pour la position de leurs travaux, se laissaient guider exclusivement par des alignements plus ou moins exacts, sans reconnaître, au moyen de galeries de recherches préalables, la nature du schiste.

Ils tombaient forcément, vu la puissance du banc, sur du rocher fournissant de l'ardoise; mais souvent après avoir fait les frais considérables du découvert que nécessite une exploitation à ciel ouvert ils finissaient par s'apercevoir que le schiste renfermait une telle proportion de pyrites de fer, que la fabrication devenait difficile et onéreuse.

Ils étaient tombés sur la pierre rude, suvvant leur expression, et devaient abandonner les travaux qui avaient causé leur ruine.

La qualité d'une ardoise peut donc être très variable.

Celle qui contient de la pyrite est altérable, parce que ce sulfure s'oxyde à l'air et devient pulvérulent; celle dont la masse est poreuse s'imprègne de l'eau des pluies et la moindre gelée suffit pour la briser. Elle a, de plus, l'inconvénient d'être perméable.

Une ardoise qui contiendrait une certaine proportion de carbonate de chaux durera peu, car l'eau désagrège lentement mais infailliblement ce sel calcaire. Les ardoises sont donc d'autant meilleures qu'elles en contiennent moins.

On peut se rendre compte de la qualité d'une ardoise au point de vue hygrométrique, par une expérience fort simple
On trouve souvent ces cristaux dans les couches grises et vertes. D'autrefois et plus fréquemment, ce sont des cristaux de pyrite de fer. Ceux-ci se rencontrent plus souvent allx affleurements qu'à une certaine profondeur.

Quand ils sont d'une ténuité extrême et pas trop abondants, ils ne nuisent pas à la qualité de l'ardoise, mais plus gros ils peuvent rendre dès son extraction, l'ardoise invendable, parce qu'ils forment des trous à sa surface et nuisent au sciage ; de plus, lorsque l'ardoise est en place ils s'oxydent, rendent la surface rugueuse, retiennent les poussières et favorisent le développement des végétaux parasites, tels que les mousses.

Il en résulte une humidité à peu près constante tout autoirs de chaque grain, qui aide à la décomposition du schiste.

De plus, une bonne ardoise doit s'altérer très difficilement à l'air et à l'humidité, pouvoir supporter des variations brusques de température, ne pas changer de couleur avec le temps Elle ne doit pas être cassante, quoique dure et inaltérable, affn qu'on puisse la travailler et la percer facilement avec la pointe du marteau de couvreur.

Les angles doivent être faciles à tailler vifs, sans quili se produise aucune exfoliation à aucun moment de son emploi.

Dans ces conditions, une ardoise à surface lisse de 3 à $4 \mathrm{~m} / \mathrm{m}$ d'épaisseur n'absorbe qu'une très farble quantité d'eau égale au plus au 0,0004 de son poids.

(i) Fuchs et Delaunay. Op. cit. 
Mais, dans une même ardoisière, on trouvera des bancs à grain plus ou moins fin, plus ou moins siliceux, donnant une ardoise plus ou moins faclle à tailler, à polir.

Ce sont toutes ces considérations qui infiueront pour la détermination à prendre sur le quantum du prix, ou sur le point d'attaque à choisir.

D'ailleurs, nous l'avons dit, il serait inutile aujourd'hui de tenter l'exploitation d'une ardoisière sans moyens mécaniques.

Ceux-ci doivent être, au contraire, des plus complets.

Ils permettront de prendre, découper, tailler les parties de schistes durs impossibles à fendre autrefois, donc jadis inutilisables, tandis que leur emploi se généralise de plus en plus. En même temps ils réduiront, dans des proportions considérables, la perte à l'abatage d'abord, à la fabrication ensuite.

La durée des ardoises est très variable et constitue une qualité de premier ordre. Celles qui sont fabriquées avec du schiste exploité à une faible distance des affleurements dirent beaucoup moins de temps que celles qui proviennent de schistes extraits à une grande profondeur.

Ceci n'est vrai qu'en général, car nous avons vu des couvertures d'ardoises de Siguer (Ariège), datant de 100 ans et qui étaient faites de simples " cosses ", c'est-à-dire avec du schiste de surface. L.es ardoises d'Angers sont moins dures que celles des Ardennes. Les premières durent de 20 à 30 ans, les secondes 100 . En revanche, le grain des ardoises ardennaises est moins fin et les couches sont moins puissantes, plus tourmentées, et à replis nombreux.

\section{VI. - TRAVAUX DE RECHERCHE ET DE MISE EN VALEUR.}

Lorsque l'on a reconnu la présence certaine, la qualité et la continuité probable d'un dépôt de schiste ardoisier; lorsque, en un mot, on veut commencer une ardoisière, on doit chercher à commaitre l'allure de la couche de schiste en faisant des travaux de recherche et en ayant égard aux accidents, tels que failles, plis, couteaux, que l'on a rencontré dans les exploitations voisines et qui peuvent se continuer quelquefois assez loin.

Il est très rare, comme nous l'avons dit, que les couches de schistes soient exploitables près des affleurements. La qualité du schiste s'améliorant généralement à mesure que la profondeur augmente, ce n'est qu'à une assez grande profondeur variant de 100 à 150 mètres que l'on doit commencer les cuvrages.

Il faut donc lorsque les couches sont inclinées, comme c'est le cas général, descendre des puits également inclinés jusqu'à une profondeur suffisante pour trouver la roche schisteuse sans ancune altération due aux agents atmosphériques ou aux effets dynamiques qui, forcément, ont plus affecté les roches de la surface.

S'll s'agit d'une ardoise d'affleurement, de schistes ardoisiers à exploiter à ciel ouvert, il y a d'abord lieu de déblayer la terrain, d'enlever les " cosses", et là on a quelquefois un nombre considérable de mètres cubes à remuer, car on reprend généralement pour les développer en les réunissant, d'anciennes petites carrières où les exploitants primitifs travaillaient morceau par morceau, sans programme, sans ordre, sans méthode, parce qu'elle leur paraissait toujours inépuisable et sur lesquelles ils laissaient tous les déblais plutôt que de dépenser du temps et de l'argent à les transporter au loin.

Cette question des déblais est de premier ordre au point de vue ardolse, puisque ainsi que nous le disions au $\$ 3$, les déblais peuvent atteindre dans certains cas jusqu'à $75 \%$ de la production.

Lorsque les points d'attaque ont été normalement choisis, que quelques petites galeries de reconnaissance ont permis de se rendre compte de la puissance et de la place du banc fissile le plus convenable, les " cosses " et déblais une fois enlevés, le carreau aplani, les voies ferrées indispensables établies, il dins 'aura plus qu'à préparer l'exploitation en carrière par gradins droits, ce qui constituera le maximum d'économie.
Si l'on peut opérer ainsi, c'est qu'on aura reconnu que le schiste est marchand dès la surface; on aura donc, de suite, une certaine production. Il faudra donc, en même temps, s'occuper des ateliers de fendage. Pour le début, ce seront de simples baraques dont les murs et le toit seront faits de débris d'ardoises qui ne manquent pas, malheureusement.

Conme toujours, nous nous montrons opposés à commencer par l'achat de machines coûteuses de sciage, fendage, polissage, et nécessitant l'établissement d'une force motrice dont l'importance relative ne peut être jamais prévue avec justesse au commencement d'une exploitation quelconque.

Mais il y auraut lieu de se préoccuper d'amener de l'eau sur le chantier de fendage des ardoises.

La pierre se fendant beaucoup mieux au sortir de l'ardoisière, lorsqu'elle est encore un peu humide que lorsqu'elle est restée un certain temps dans un endroit sec, il est nécessaire d'entretenir l'humidité en recouvrant les tranches des clalles ivec de la terre glaise qu'on arrose fréquemment.

Pour ce motif, il sera toujours bon d'organiser ses équipes à l'abatage et au fendage, de manière à ce que les fendeurs n'aient que peu de schiste abattu d'avance.

Mais ce cas d'exploitation à ciel ouvert si pratique, si peu :her est, pour ainsi dire, un rêve. Presque toujours on aura affaire à une conche souterraine.

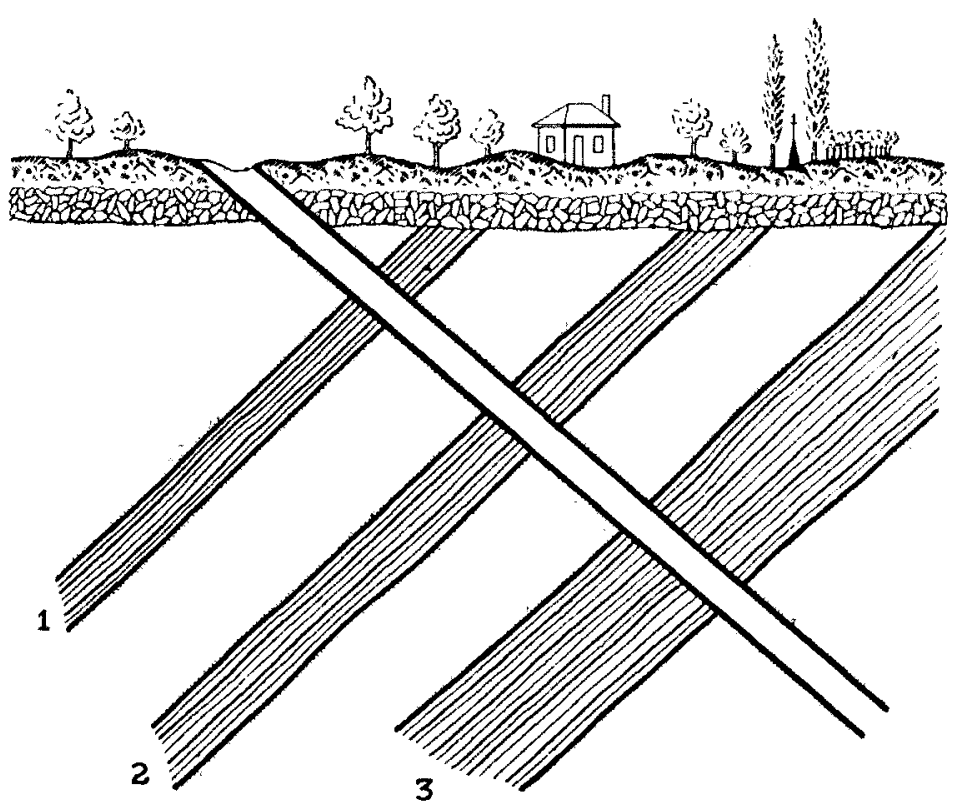

Fig. r. - Puissance du terrain ardoisier et disposition des bancs utulisables

On aura alors à opérer par puits, descenderies ou traversbancs qu'on dirigera, pour ces derniers, perpendiculairement a la direction des bancs ardoisiers ou s'il s'agit de descendre plus fréquemment dans la couche même, en direction, pour exploiter ensuite par ouvrages, gradins droits ou renversés.

S'il s'agit d'une exploitation par puits (extrêmement rare), c'est qu'on aura préalablement reconnu toutes les données nécessaires par des sondages, ainsi que nous le disons plus loin.

Si l'on opère par descenderies ou travers-bancs, ce sera également après que d'autres observations auront permis de reconnaître, préalablement, la présence du dépôt ardoisier.

En ce cas, le travers-bancs ou la descenderie constitueront tout d'abord une véritable galerie de recherches, car ils recouperont tous les bancs ardoisiers ou non et permettront de se rendre un compte exact de l'épaisseur, de la disposition et du nombre réel de bancs intercalaires, de schistes stériles (non ardoisiers) et de la qualité de l'ardoise. En tout état de cause, ce ne seront donc jamais des travaux en pure perte.

Leur emplacement devra être choisi de telle sorte que les déblais puissent ne pas être encombrants ni onéreux. Il ne faut pas oublier qu'ils sont considérables. 
S'il s'agit de recouper en profondeur différents bancs dont on connaît plus ou moins bien l'affleurement, on pourra avoir une idée à peu près exacte sur la dimension à donner au travers-bancs; on saura combien de couches on doit rencontrer et à peu près à quelle distance on doit couper les bancs ardoisiers fissiles.

On pourra, dans ce cas, afin de bien se rendre compte de la composition du sous-sol ardoisier, pratiquer une descenderie perpendiculairement aux couches. Cette manière d'opérer lorsque les circonstances ne la rendent pas onéreuse, est très commode pour apprécier le nombre, la puissance et la disposition des couches I, 2, 3, etc. (fig. I).

Un puits vertıcal donnera les mêmes résultats, mais il faudra par des recoupes vérifier l'inclinaison des couches, sans quoi, le puits les recoupant en brais, on prendrait la longueur d'une diagonale au lieu de celle d'une perpendiculaire pour la puissance.

De mêrne, comme à la surface il y a toujours des éboulis, des dépôts sur les pentes, en quantité plus ou moins importante et qui peuvent atteindre 1o et 20 mètres de hauteur, ir importe de choisır un point où le sol s'élève rapidement pour avoir plus vile de la véritable ardoise de profondeur et ne pas occasionner de perturbatıons à la surface.

Bien souvent, en effet, on n'achète ou on ne loue que le droit d'exploitation du tréfonds.

Il n'est pas toujours possible de creuser une galerie inclinée partant des affleurements et suivant la pente de la couche. Parfois les limites de la concession ou l'obligation de profiter d'un chantier bien disposé pour les clépôts d'ardoises, oblige la Société exploitante à établir, à travers bancs, un puits vertical ou une galerie ayant une inchnaison contraire à celle de la couche. Lorsqu'on atteint celle-ci, on change alors l'inclinatson de la galerie pour suivre celle de la couche. Mais les galeries à travers-bancs et les puits verticaux sont très cô̂uteux. Les derniers, principalement, nécessitent l'emploi d'un matérial spécial. Ils amènent dans les ouvrages les eaux qui suivent les bancs et les fissures,et ils augmentent les frais d'extraction en nécessitant l'emploi d'hommes à poste fixe aux changements de direction.

S'il s'agit d'une reprise d'ancienne ardoisière, ce sera bien rare si les plans existants seront compréhensibles et donneront les cotes et l'emplacement des ouvrages des piliers et des galeries. Bien plus rarement encore (Jamais) on n'aura pas à épuiser. En ce cas, l'achat d'une chaudière et de pompes s'impose ou bien d'une locomobile qui servira en même temps à actionner lc treuil nécessaire à la remonte des déblais encombrant l'ardoisière abandonnée. On peut avoir des surprises bien désagréables et l'on fera bien, dans les prévisions de tripler ou quadrupler les chiffres rationnels relatifs à tout, mais surtout à l'épuisement, car fatalement on aura soit à épuiser des ardoisières voisines également abandonnées, soit à assécher des nappes d'infiltration dont aucune donnée ne peut déterminer l'importance.

S'il y a déjà des travaux faits, par exemple dans le cas d'une descenderie en pleın schiste ardoisier, comme on le fait généralement dans l'Est de la France, on aurait grand interêt à mener le plus bas possible, dans ces travaux, une petite galerie horizontale.

Plus on pourratt commencer cette galerie loin de la surface et mieux cela vaudrait. Elle permettra de reconnaître si l'on est bien dans la veine,et même en poussant assez loin des deux côtés, survant les cas, s'il ne se trouve pas dans le voisinage d'autre veine exploitable.

Il arrive en effet souvent que l'on se trouve dans un massif ardoisier dont le caractère schisteux est très développé.

C'est très rare qu'll n'y att qu'une veine, mais aussi, précisément à cause de ce caractère schisteux, très visible en surface, on peut être induit en erreur sur la qualité de l'ardoise que l'on suppose exister en dessous.

Certaines parties de l'affleurement peuvent donner lieu aux plus belles espérances et faire croire avec la plus grande facihité à l'espérance d'une veine qui deviendra exploitable à une certaine distance. Il est donc très rationnel de commencer les travaux dans cette veine et de la mieux reconnaitre un peu plus bas.

Presque tout de suite, en dessous de la surface, on trouve alurs comme nous l'avons déjà expliqué, les lits de schistes, très fissurés, très fracturés, cassés en petits morceaux et comme emplés les uns au-dessus des autres, sans aucun ordre.

C'est naturcllement presque toujours l'affleurement de ces schistes, plus ou moins ardoisiers, qui a déterminé les premiers occupants à chercher plus bas les véritables bancs d'ardoises.

En dessous de cet affleurement (qui lui-même est, dans la plupart des cas, recouvert d'une sorte de terre végétale $A$, formée de débris très fins de schiste) la succession des assises présente un aspect semblable à celui de la figure 2 .

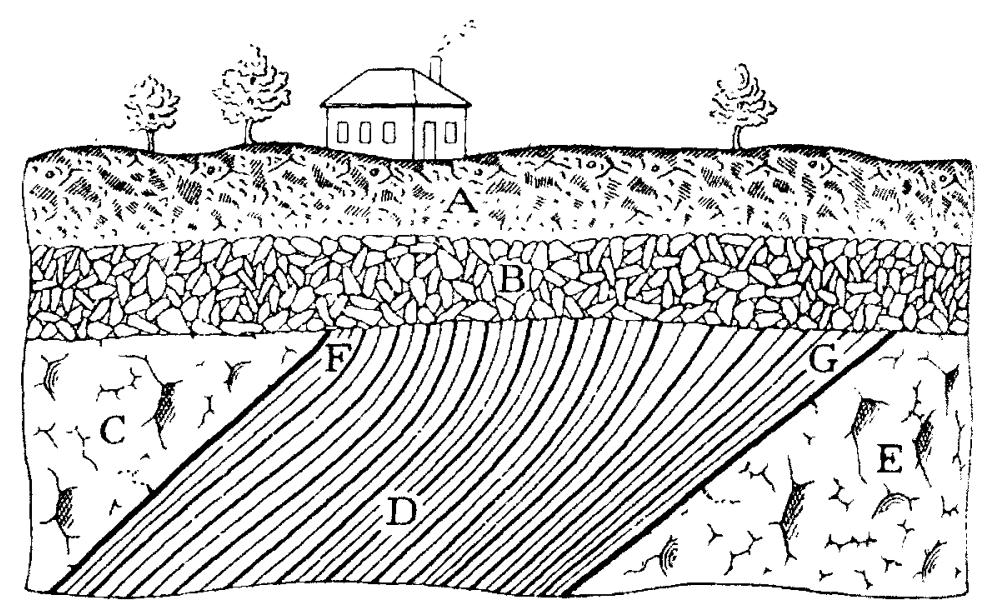

Fig. 2. - Disposition des schistes ardoisiers.

On trouve d'abord:une couche $\mathrm{B}$ de débris plus gros et dis. posés avec le plus grand désordre, comme s'ils constituaient un remblai fait à la brouette, puis, généralement avec une démarcation très nette et en stratification absolument discordante la couche C D E indiquant nettement que la partie supérieure du pli déjà cassée probablement et délitée par le soulèvement, a disparu entièrement par les érosions postérieures.

A la surface d'arrachement du pli, les schistes sont fendillés à l'extrême, découpés en minces plaquettes et brisés en menus morceaux.

A mesure que l'on s'enfonce, l'effort auquel ils ont été soutmis étant de moins en moins considérable, ils se présentent moins fissurés, moins cassés, pour devenir même, à un certain moment, comparts. On a souvent alors une veine schisteuse, telle que $D$ entre deux veines de grès $E$.

Lorsqu'une telle veine $D$ apparait en surface, on peut avoir intérêt à y pousser une descenderie, mais il n'est pas toujours facile de reconnaitre dans quelle partie de $F G$, il est le plus avantageux de le faire.

Quelquefois cette veine schisteuse $D$ peut être très puissante et contenir des interstratifications de grès. Elle peut de $F$ en $G$ présenter une ou plusieurs couches de schiste ardoisier qui, à une certaine profondeur, deviendra exploitable ; c'est donc dans une de ces veines qu'il faut percer la descenderie, en ayant soin, quelle que soit la puissance de la veine ardoisiète, de se placer au mur.

Les vieux ouvriers mineurs des Ardennes, très infatués de leur métier et dont il est matériellement impossible d'ouvir l'intelligence à la moindre méthode nouvelle ou simplement rationnelle d'exploitation, prétendent le contraire, en objectant qu'en prenant le toit de la veine pour toit de la descenderie, ce dernier est beaucoup plus solide. Cela n'est vrai que d'une manière relative.

Il suffit, en effet, d'avoir soin de prendre comme toit de la descenderie un avantage de la veine, bien choisi, c'est-d̀-dire, 
une de ces sortes de plan de clivage du schiste qu'on rencontre fréquemment et formant un délit naturel.

Ce sont ces avantages qui constituent les différents lits d'ardoises

Dans une même veine, certains constituent un toit tout aussi solide que le toit naturel et la plupart le sont plus que suffisamment pour résister à toutes les poussées possibles, surtout arec un vide de 3 ou 4 mètres seulement.

Par conséquent, il n'y a aucun inconvénient à les employer. La descenderie étant placée au mur, on pourra attaquer ensurte des ouvrages ou des galeries pour l'exploitation par gradins de bas en haut. Il est évident qu'il est alors plus facile de commencer le crabotage, l'exploitation des ouvrages ou toute autre méthode d'exploitation. Un argument plus sérieux serait qu'au point de vue des épuisements, il est nécessaire d'avoir des puisards à plusieurs niveaux et que, par conséquent, on doit toujours creuser la galerie inclinée à une certaine hauteur au-dessus du mur. Sauf en quelques cas particuhers, 1 nous paraît bien préférable de crever au besoin le mur pour faire ces puisards, et si les roches rencontrées sont perméables, de les recouvrur d'une petite chape en ciment.

L'Ingénieur chargé de la mise en valeur d'une ardoisière de ce genre aura à lutter contre le mauvais vouloir de ses ouvners d'abord, et aussi contre les difficultés d'ordre pratique el technique provenant de la comnaissance insuffisante des veines.

C'est pour cela qu'après s'être avancé, plus ou moins, selon la qualité du schiste reconnu, on aura intérêt à modifier peuttre la pente de la descenderie pour se placer plus bas ou plus haut selon la disposition, la qualité et la puissance de la veine. C'est alors qu'une précieuse indication peut être fournie par la petite galerie horizontale, dont nous avons parlé pius haut.

Cette galerie A B (fig. 3 ) permettra à l'ingénieur de connaître la composition des dépôts schisteux dans toute la puissance de la veine et lui montrera, par exemple, si la descenderie doit être continuée avec la même pente, en ligne droite, ou bien s'il faut changer la pente, ou bien encore s'il est préférable de se mettre en $D^{\prime}$, par exemple, et de se raccorder en D par une portion de voie moins inclinée.

Ces questions sont à élucider dès le début de la mise en valeur, parce qu'elles jouent un rôle prépondérant dans l'extraction future.

Dans le même ordre d'idées, le treuil doit être étudié pour une exploitation jusqu'à une profondeur déterminée. Suivant le cas, il sera à vapeur ou à l'électricité, ce dernier système devant être préféré toutes les fois qu'il sera possible, à cause de son extrême simplicité, de sa robustesse, de son peu d'entretien et de sa facilité d'emploi.

De même, la voie et les wagonnets demandent une étude spéciale et diffèrent un peu des autres exploitations minières.

La voie doit être robuste; plus elle sera large mieux cela vaudra. Celle de 80 centim. est fort pratique. On devra placer, parallèlement à chaque rail ou dans le milieu, de fortes crémaillères destinées à retenir la bêche dont sont munis les wagonnets, et qui viendrait s'intercaler dans une dent, en cas de rupture du câble. On aura le plus grand intérêt à avoir des wagonnets en bois, deux ou trois fois plus longs que larges; ils sont bien préférables aux wagonnets en tôle.

L'épuisement d'une ardoisière est égaleruent un des problèmes principaux de sa mise en valeur et nous insistons d'une manière spéciale sur les appareils à employer au paragra-
phe 8 .

Quant à l'installation et au type de treuil, c'est une question éminemment variable. Nous préconisons fort un type analogue à ceux dont il est parlé au dit paragraphe 8; mais en tous cas, toutes les fois que d'autres difficultés d'ordre plus considérable ne viendront pas l'empêcher, il faudra installer ce treuil le plus haut possible au-dessus du sol. Il n'est, en effet, pas plus coûteux de remónter les wagonnets de quel- ques mètres, ce qui constitue un tantième insignifiant de la profondeur de la descenderie et l'on se donne, par ce moyen, la facilité d'avoir une hauteur beaucoup plus considérable à utiliser pour les déblais sans être obligé, par la suite, de les remonter soit par des voies ferrées, soit à la brouette.

$\mathrm{Si}$, au contraire, il s'agit d'ouvrir une ardoisière souterraine nouvclle, on commencera par faire plusieurs sondages ou puits pour reconnaitre exactement l'affleurement de la couche

On établira une galerie principale suvant la ligne de plus grande pente en s'écartant le plus possible des forts plis ou parties mauvaises que l'on est susceptible de rencontrer, et en ayant égard à l'emplacement qui doit être utilisé par la machine à vapeur et les ateliers de fabricatıon près de l'entrée de la galerie.

Les débris d'ardoises sont irès encombrants, ainsi que nous l'avons dit, et l'on est souvent gêné pour les loger. Il faut donc acheter, par avance, des terrains morts dans les environs mmédiats des travatix.

Quant à la galeric elle-même, il faut lui donner une largeur de $2 \mathrm{~m}$. 50 à 3 mètres pour y établir une voie ferrée, les pompes et un passage pour l'ouvrier qui doit assurer le graissage des galeis qui supportent les tirants des pompes, ou surveiller les conduites de vapeur, la voie, etc. Ce passage, d'ailleurs, sera utilisé ensuite pendant les premières rannées d'exploitation pour la circulation des ourriers de fond.

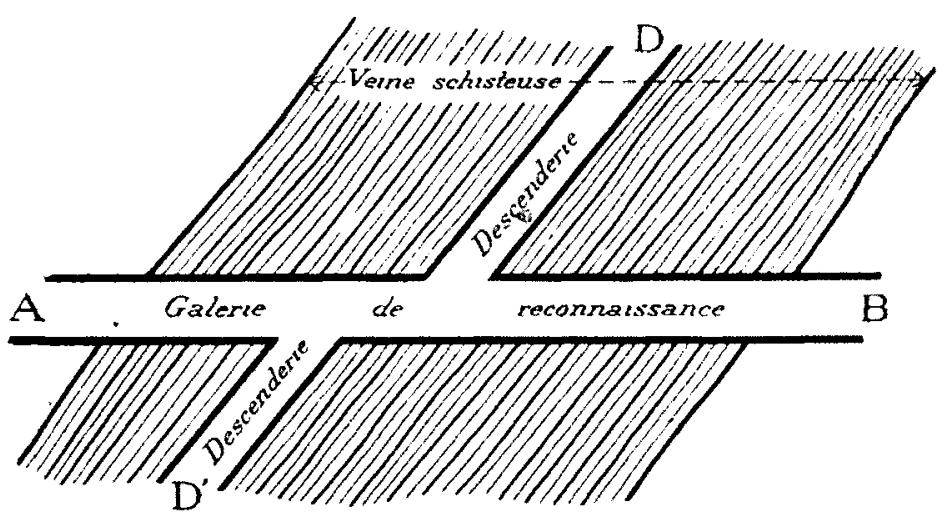

Fig. 3. - Descenderie en pleine veine.

L.a galerie doit avoir la plus grande longueur possible et au moins 200 mètres, de façon à attaquer immédiatement la couche à une très grande profondeur, c'est-à-dire vers l'endroit où l'on a le plus de chances de rencontrer du schiste de très bonne qualité.

On créera sur un côté de la galerie des réservoirs d'eau ou puisards dans lesquels les pompes qui seront étagées enver. ront les eaux destinées à être enlevées à la surface. Le fond de la galerie devra être à une certaine hauteur au-dessus du fond de la couche, de façon à conserver des réservoirs intacts lorsqi:e l'on fera l'exploitation des ouvrages. On établira ensuite. à partir de la galerie principale, une galerie en direction sur chacun des côtés pour reconnaître la nature de la couche. L'aérage de ces galeries devra être assuré au moyen d'un ventilateur (I).

Il est évident que pour tous ces travaux on aura besoin de force motrice; donc l'achat d'une chaudière et d'une machine à vapeur s'impose, à moins que l'on ne trouve à utiliser une chute d'eau avec un petit barrage défnitif ou de fortune.

On opérerait alors électriquement, ce qui vaudrait mieux. Quoiqu'il en soit, dès que les puits inclinés seront arrivés dans la bonne ardoise fissile, on préparera des ouvrages de chaque côté, soit d'après l'antique méthode des Ardennes, décrite dans tous les cours d'exploitation, soit à l'aide de perforatrices, système que nous ne saurions trop préconiser.

Le matériel nécessaire aux travaux de fond, pour la mise

(1) Watrin. Op. cit. 
en valeur surtout, est très restreint : il se compose de quelques barres à mine, de massettes, de scies et de longs couteaux d'acier pour la division des plaques, enfin de quelques wagonnets.

Le plus souvent, au heu de ménager des réservoirs d'eau sur les côtés, comme nous l'avons dit plus haut,il sera plus simple de ménager au bas de la galerie maîtresse un puisard dans lequel on placera un pulsomètre ou roieux, si possible, une poinpe électrique pour l'exhaure de l'eau.

En résumé, par ces galerıes de profondeur, suivant la pente de la couche reconnue en direction, on se rendra compte de l'importance el de la qualité du gisemẹnt en le préparant pour l'exploitation.

En même temps, il sera bon d'opérer quelques sondages destinés à reconnaître la place, l'épaisseur, la nature et le nombre des couches de schiste ardoisier.

En surface, on aménagera très modestement une baraque pour les fendeurs en utilisant, autant que possible, les déchets d'ardoise pour la construction.

\section{VII}

11 nous paraît inutile de nous étendre beaucoup sur l'exploitution des ardoisières, par suite des descriptions très complètes qui en ont été donnćes dans les ouvrages spéciaux.

D'ailleurs, l'exploitation de l'ardoise quoique très spéciale, est des plus simples. Seule, parmi toute l'industrie minière, elle s'est confinée dans une routine désolante et c'est à peine si les progrès immenses de l'outillage moderic commencent à s'introduire chez les ardoisiers.

Mais nous allons d'ici peu assister à un réveil qui sera formidable, parce qualors on changera tout.

L'antique crabotage et les ouvrages auront vécu... et ce ne sera pas trop tôt.

Disons donc simplement quelques mols de ces procédés qui sont encore employés partout.

Très anciennement, les ardoisières étaient exploitées en laissant, entre les ouvrages qui avaient une largeur à pèu p̉ès égale à la longueur, des piliers disposés assez irrégulièrement et autant que possible en quinconce. Dans une exploitation d'une certaine importance, le plan des piliers et des ouvrages ressemblait à un vaste échiquier.

Celte méthode consistait à établir, sur toute l'épaisseur de la couche exploitable, des ouvrages ayant vingt à trente mètres de longueur, qui étaient séparés par des piliers dont la longueur et la largeur variaient dé six à dix mètres, et dont les faces étaient perpendiculaires au plan des couches. Les piliers n'étaient pas toujours disposés d'une manière régulière, on les éloignait plus ou moins, suivant leur solidité, ou suivant les dangers d'éboulements; leurs dimensions variaient avec les épaisseurs des couches à exploiter.

On conçoit facilement qu'avec des piliers disposés en quinconce, il eût été impossible d'établir des voies ferrées, à travers les ouvrages, pour le transport de la pierre. Les ouvriers devaient faire tous les transports à dos, ou tout au moins jusqu'à une galerie inclinée, ce qui rendait l'exploitation fort cô̂teuse.

Les piliers étant disposés normalement à la couche et n'étant pas reliés entre eux, finissaient par se rompre sous le poids du loit. En outre, il fallait creuser à grands frais des puisards spéciaux destinés à retenir les eaux pendant un certain temps. Toutes ces considérations ont déterminé les exploitants à abandonner cette méthode pour la remplacer par une autre qui est beaucoup plus avantageuse.

Elle consiste, une fois la descenderie faite suivant la ligne de plus grande pente de la couche, à pratiquer de vastes excavations nommées ouvrages que l'on creuse de chaque côté de la galerie principale servant à l'extraction de la pierre. Les cuvrages ont généralement I 5 mètres de longueur suivant la pente; ils sont séparés par des piliers de 4 à 5 mètres de longueur ou épaisseur.

La largeur des ouvrages n'a pour limites que la fin de la couche des parties inexploitables ou les limites de la concession. La hauteur des excavations est égale à l'épaisseur de la couche exploitable. Les piliers ont à peu près la même direction que la couche; ils sont percés de distance en distance pour l'aérage des ouvrages, le passage des ouvriers et l'écoulement des eaux. Un donne le nom d'airures à ces percées, qui ont de 4 à 8 mètres de largeur.

Généralement, il n'y a qu'un pilier sur 2 dans lequel il y a des atrures, de façon à ce que l'autre pilier, nommé naie, puisse retenur les eaux et les conduire dans un pusard creusé à proxi. mité de la galene principale.

Les 2 faces des piliers doivent être dressés au pic et être parallèles, de façon à leur conserver une épaisseur uniforme.

Les piliers doivent eux-mêmes avoir des directions parallèles, pour que les ouvrages aient des dimensions régulières. On doit, autant que possible, conserver comme piliers les parties de la couche impropres à la fabrication des ardoises.

On donne les noms de toit et de mur aux faces de l'ouvrage qui se rapprochent le plus du toit et du mur de la couche. Le côté droit est celui que l'on a, à sa droite, en suivant la pente de la galerie inclinée. Le côté gauche est celui qui est opposé. Le derrière d'un ouvrage est la face contre laquelle les eanx coulent naturellement, le devant est la face qui lui est opposée. Le devant et le derrière d'un ouvrage et, par conséquent, les faces corresponidantes des piliers, sont à peu près perpendiculaires au mur et au toit de la couche.

L'exploitation des ouvrages comprendi deux phases bien distinctes : le crabotage ou havage, et l'abatage de la pierre.

Le crabotage consiste à préparer une excavation ayant yo à 80 centim. de hauteur, la même longueur que l'ouvrage, et une largeur qui varie avec la résistance de la couche.

L'abatage de la pierre peut être fait de deux manières:

$I^{\circ}$ Par la méthode en rehaussant, c'est-à-dire en se dirigeant du mur vers le toit.

$2^{\circ}$ Par la méthode en abaissant, c'est-à-dire en se dirigeant du toit vers le mur (I).

(A suivre).

M. Leconte-DeNis, Ingénieur civil des Mines.

\section{La Houille blanche en Norvège}

Nous mettons sous les yeux de nos lecteurs la communication suivante qui nous a été adressée par un de nos abonnés, industriel à Christiania; persuadés que les documents qu'elle contient les intét. ressera; nous les donnons tels gu'ils nous sont communiqués par l'auteur.

Considérations générales. - Maintenant que l'Electricité joue un si grand rôle dans toutes les industries modernes, il y a pour cellés' qui existent dans les pays riches en forces hydrauliques un avenir de prospérité sur lequel on ne saurait trop appeler l'attention.

Or, parmi nos pays d'Europe, la Norvège se place au premier rang sous ce rapport de l'évolution provoquée par la houille blanche dans le plus grand nombre de nos indus. tries. Cette situation privilégiée est due aux circonstances suivantes:

$I^{0}$ Il y a en Norvège de nombreuses et puissantes chutes d'eau susceptibles de mettre en œurre une quantité d'énergie considérable. Ces chutes sont presque toutes d'appro' priation très facile. En général elles sont situées à peu de distance de la mer, en sorte que leur puissance peur être transmise économiquement à des entreprises industrielles

(I) Watrin. Op. cit. 